\title{
A supernumerary marker chromosome originating from two different regions of chromosome 18
}

\author{
Benno Röthlisberger, Krystyna Chrzanowska, Damina Balmer, Mariluce Riegel, \\ Albert Schinzel
}

\begin{abstract}
By random amplification of a microdissected chromosome using the degenerate oligonucleotide primed polymerase chain reaction (DOP-PCR) and forward painting (microFISH), we characterised an extra structurally abnormal chromosome (ESAC) or supernumerary marker chromosome in a mentally retarded girl with a pattern of dysmorphic features. It could be clearly shown that the small marker chromosome originates from two different regions of chromosome 18, $18 \mathrm{p} 11.1 \rightarrow 18 \mathrm{q} 11.1$ and $18 \mathrm{q} 12.3 \rightarrow 18 \mathrm{q} 21.1$ respectively. Maternal origin of the de novo ESAC and biparental origin of the normal homologues of chromosome 18 were shown by PCR of several highly polymorphic microsatellites. In this case, application of microFISH was a prerequisite for rapid and precise characterisation of an ESAC. A definite identification of this discontinuous supernumerary marker chromosome would not have been possible using FISH with centromere specific probes or multicolour FISH approaches. (f Med Genet 2000;37:121-124)
\end{abstract}

Keywords: supernumerary marker chromosome; microdissection; FISH; chromosome 18

At least 168 cases with an ESAC (excluding those from chromosome 15) originating from all chromosomes have been documented. ${ }^{12}$ Birth prevalence is estimated at 0.14 to 0.72 per $1000 .{ }^{3}$ Despite the relatively high frequency of trisomy 18 detected pre- and postnatally, only four cases with a marker chromosome derived from chromosome 18 have been reported.

Subjects with a supernumerary marker chromosome have a duplication and in some cases a triplication of the material comprising the ESAC. The risk of an abnormal phenotype associated with a randomly ascertained de novo ESAC derived from acrocentric autosomes (excluding chromosome 15) is approximately $7 \%$, compared with approximately $28 \%$ for ESACs derived from non-acrocentric autosomes. ${ }^{1}$ The great variability of clinical symptoms in patients with ESACs of the same chromosomal origin is probably the result of variations in the genetic content, the degree of mosaicism, and uniparental disomy of the normal homologues from which the ESACs are derived. MicroFISH, which gives detailed information about the chromosomal content of the ESAC, is the best way to characterise a supernumerary marker chromosome. Not only is/are the chromosome(s) involved identified, but in addition the exact segment(s) of the latter can be clarified. The importance of investigating large ESACs by microFISH has been shown by Blennow et $a l^{4}{ }^{4}$ who described a marker originating from three different chromosomes. However, the origin of most supernumerary marker chromosomes is determined by FISH analysis using a centromere specific probe or a chromosome specific library or both. This is generally considered to be sufficient if the marker chromosome is very small, since the common opinion is that the marker contains only centromeric and pericentromeric sequences. However, as we show in a case with an ESAC originating from chromosome 18 , this assumption is inadequate, since even smaller markers may be the product of more than one chromosome region.

\section{Case report}

This 13 year old girl is the second child of healthy, unrelated parents. At birth the father was 25 years old and the mother was 24 years old. The girl was born at 42 weeks of gestation after an uneventful pregnancy. Birth weight was $3600 \mathrm{~g}$ (50th centile), length was $56 \mathrm{~cm}$ (>90th centile), and occipitofrontal head circumference (OFC) was $35 \mathrm{~cm}$ (between the 25 th and 50th centile). Clinical examination at the age of $11 \frac{1}{2}$ years showed height $147 \mathrm{~cm}$, weight $39.8 \mathrm{~kg}$, and OFC $52 \mathrm{~cm}$, all within the normal range. Total hand length $(18.2 \mathrm{~cm})$ was on the 10th centile and middle finger length $(7.0 \mathrm{~cm})$ was on the $3 \mathrm{rd}$ centile. The girl displayed the following anomalies: hypertelorism with inner canthal distance $(3.7 \mathrm{~cm})$ and outer canthal distance $(9.8 \mathrm{~cm})$ above the 97 th centile, bilateral epicanthic folds, upward slanting palpebral fissures, short and upturned nose, long philtrum, short neck, widely spaced nipples, short 5th fingers, cubitus valgus on both sides, and three hyperpigmented areas over the abdomen (fig 1). Pubertal development was at Tanner stage 3 .

The patient's psychomotor development was moderately retarded with a global IQ of 76 . She started to walk at 10 months of age and spoke her first single words at 3 years; she is hyperactive and suffers from dyslalia.

\section{Methods}

Metaphase chromosome preparations were obtained from PHA stimulated lymphocyte cultures from the patient and both parents according to standard procedures. GTG banded chromosome preparations were examined. 


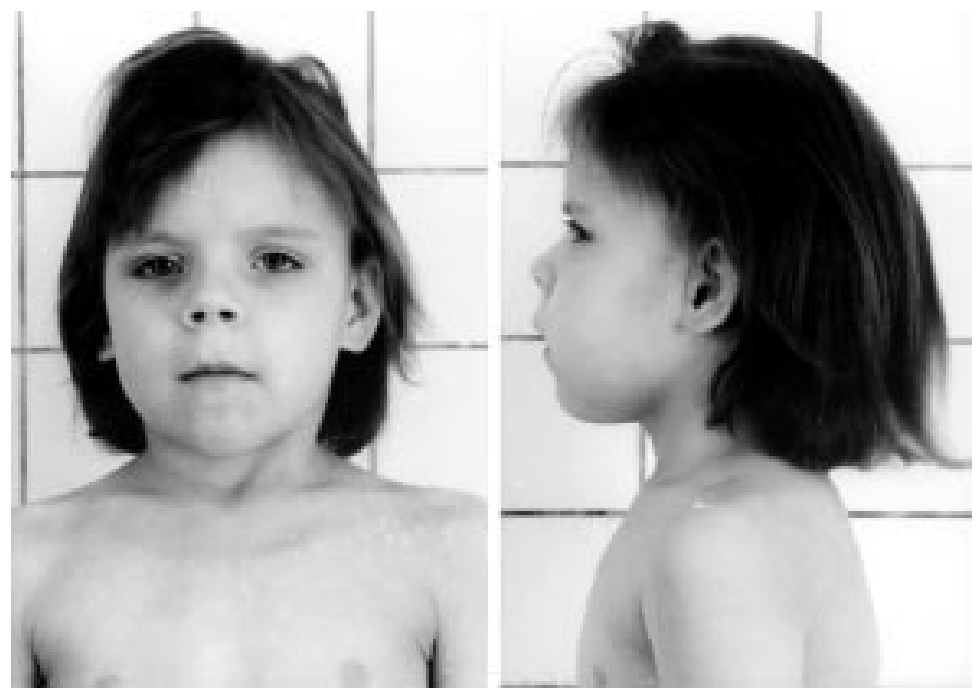

Figure 1 Face of the patient at the age of 7 years. Note hypertelorism, bilateral epicanthic folds, short and upturned nose, and long philtrum. (Photographs reproduced with permission.)

Chromosome microdissection was performed according to a slightly modified protocol by Senger et al..$^{5}$ Five ESACs were transferred to a $2 \mu \mathrm{l}$ collection drop, then amplified by DOP-PCR, and labelled with biotin.

FISH analysis was performed according to Lichter et $a l^{6}$ using the specific chromosome library generated by microFISH. The hybridised metaphases were examined with a Zeiss Axioplan epifluorescence microscope. Subsequent analyses were performed by image acquisition using a CCD camera and digital image analysis (QUIPSTM, VYSIS).

To determine the origin and formation of the supernumerary chromosome, genomic DNA from the patient and both parents was amplified by standard PCR with commercially available highly polymorphic microsatellites (Research Genetics ${ }^{\circledR}$ ), loaded onto a $6 \%$ polyacrylamide/urea gel, and visualised by silver staining.

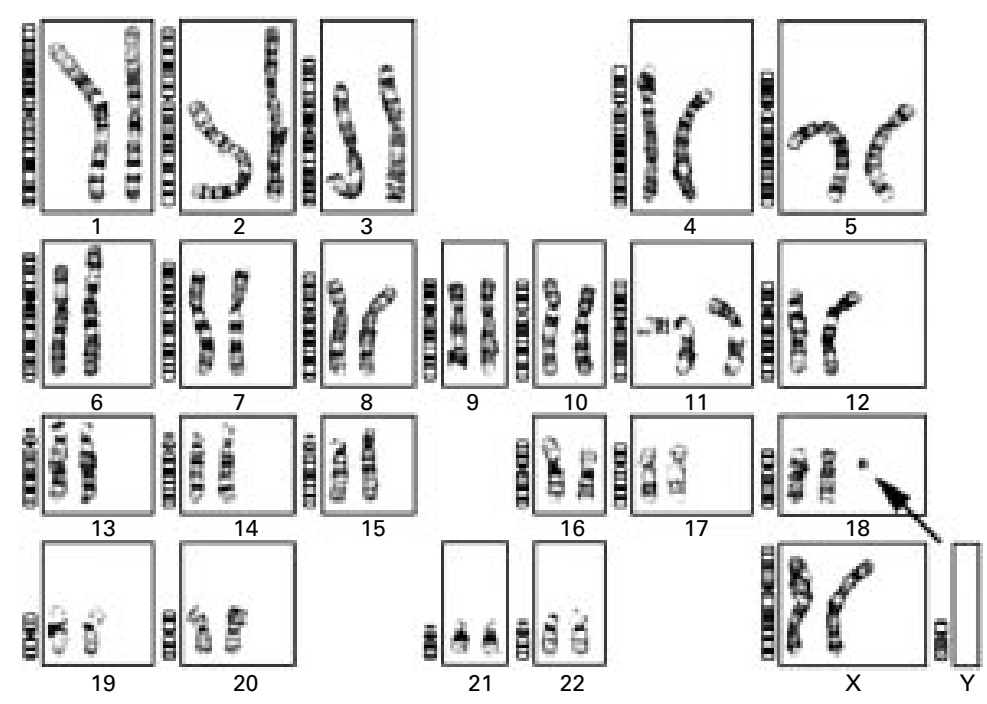

Figure 2 GTG banded karyotype of the patient. Arrow points to the supernumerary marker chromosome.

\section{Results}

Chromosome preparations from blood lymphocyte cultures of the patient showed an additional small marker chromosome in $70 \%$ of the metaphases investigated (fig 2). Chromosome analysis of the mother and the father showed normal karyotypes. In order to determine the origin of the ESAC, two completely independent microFISH experiments with reverse painting on normal metaphases were performed. It could be seen that the ESAC was formed by two regions of chromosome $18,18 \mathrm{p} 11.1 \rightarrow 18 \mathrm{q} 11.1$ and $18 \mathrm{q} 12.3 \rightarrow 18 \mathrm{q} 21.1$ (fig 3 ). The presence of chromosome 18 specific alphoid centromeric sequences was confirmed by FISH analysis with a biotinylated centromere specific probe (D18Z1). Following the detailed system of the ISCN $^{7}$ the aberration can be written as: $46, \mathrm{XX}[30 \%] / 47, \mathrm{XX},+\operatorname{mar}[70 \%] . \mathrm{rev}$ ish der $(18)(: p 11.1 \rightarrow \mathrm{q} 11.1:: \mathrm{q} 12.3 \rightarrow \mathrm{q} 21.1:)$ de novo. It should be mentioned that the orientation of the segment $18(\mathrm{q} 12.3 \rightarrow \mathrm{q} 21.1)$ is not clear.

For determination of the parental origin of the de novo ESAC and confirmation of the breakpoints, microsatellite analysis was performed on genomic DNA. Owing to the patient's relatively high percentage of cells with the marker chromosome, the microsatellite analysis resulted in clear differences in the intensities of the maternal and paternal bands. All informative markers mapping to the duplicated regions were consistent with a maternal origin of the intenser band, whereas markers lying outside the duplicated regions resulted in similar intensities of the maternal and paternal bands and thus were in agreement with biparental inheritance of the normal homologues of chromosome 18 (table 1 ).

To exclude a paracentric inversion or an intrachromosomal insertion of the long arm of chromosome 18 in one of the parents, a reverse painting experiment on metaphases of both parents using the library created by microdissection of the ESAC was performed. The signals detected were identical to the signals detected on the control slide. Thus, a cytogenetically undetectable rearrangement in the relevant region of the long arm of chromosome 18 in one of the parents, namely the mother, could be excluded.

\section{Discussion}

While classical cytogenetic procedures (G, Q, $\mathrm{C}$ banding, silver staining) can provide information regarding the structure of a marker, it is limited in its power to identify the origin of marker chromosomes. Molecular cytogenetic techniques, namely FISH, have provided a valuable method for characterising the majority of ESACs. Different types of probes are available for this use. Since it is generally assumed that ESACs usually derive from a single autosome, originating from the centromeric region and eventually from adjacent pericentric regions, most of the ESACs have been characterised by FISH studies using centromere specific probes. Nevertheless, at least 10 cases have been published in which the chromosomal origin could not be determined using alphoid repeats. ${ }^{1}$ In two reports, more complex ESACs 


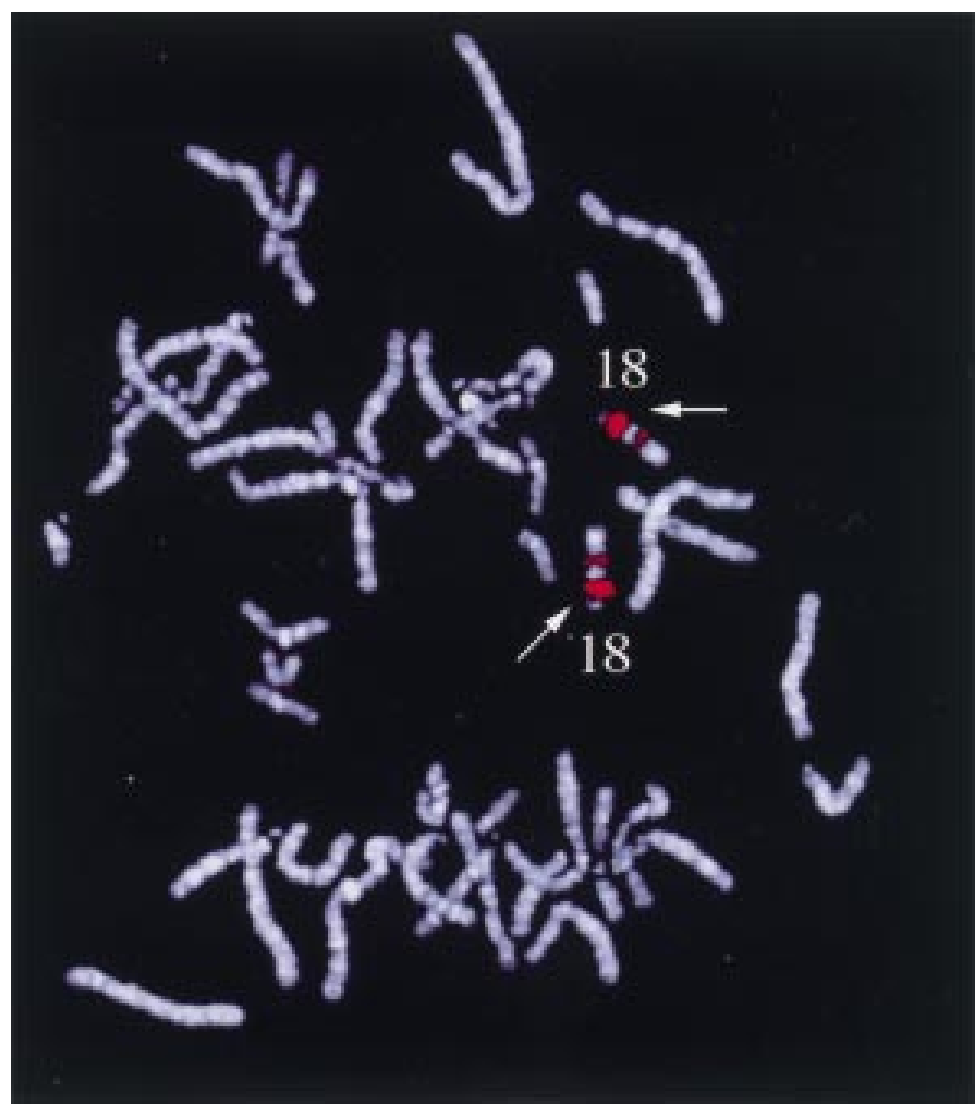

Figure 3 Forward painting using the probe generated from DNA obtained by microdissection of the supernumerary marker chromosome. Note the two signals, one centromeric (indicated with arrows) and one on the long arm of chromosome 18.

Table 1 Results obtained by microsatellite analysis

\begin{tabular}{llll}
\hline Primer & Locus & $c M$ & Alleles \\
\hline D18S71 & 18p11.23p11.22 & 47 & $\mathrm{bc}, \mathrm{bd}, \mathrm{ac}$ \\
D18S1104 & $18 p 11.1 q 11.2$ & 52.8 & $a \mathbf{b}, b d, a c$ \\
D18S869 & $18 \mathrm{q} 11.1 \mathrm{q} 11.2$ & 52.2 & $\mathrm{bc}, \mathrm{bc}, \mathrm{ab}$ \\
D18S1143 & 18 & 66.7 & $\mathrm{bc}, \mathrm{bd}, \mathrm{ac}$ \\
$D 18 S 1111$ & $18 q 21.1 q 21.1$ & 67.09 & $\mathbf{a} b, a c, b b$ \\
$D 18 S 455$ & $18 q 21.1 q 21.1$ & 68.6 & $\mathbf{a} b, a a, b b$ \\
$D 18 S 473$ & $18 q 21.1 q 21.1$ & 71.42 & $\mathbf{a} b, a b, b b$ \\
$D 18 S 474$ & $18 q 21.1 q 21.1$ & 71.3 & $\mathbf{c} d, b c, a d$ \\
D18S35 & $18 \mathrm{q} 21.2 \mathrm{q} 21.2$ & 78.54 & $\mathrm{ac}, \mathrm{ab}, \mathrm{cc}$ \\
\hline
\end{tabular}

The alleles are given in the order patient, mother, father. The markers showing intenser maternal alleles in the patient are given in italics and the intenser alleles themselves in bold.

Allele designations (a to d) are arbitrary. Genetic mapping according to the sex averaged linkage map by the Genetic Location Database (LDB).

originating from different chromosomal regions have been described. In one report the (large) ESAC was shown to contain sequences from the centromeric part of chromosome 7, the telomeric region of the long arm of chromosome 5, and the telomeric region of the short arm of the $\mathrm{X}$ chromosome. ${ }^{4} \mathrm{~A}$ second one, in this case a small ring, was shown to be derived from chromosome 4 , having a complex composition including centromeric, pericentromeric, and $4 \mathrm{q} 31.1 \rightarrow \mathrm{q} 31.3$ sequences. $^{8}$ The accurate characterisation of these discontinuous marker chromosomes was only possible using the microdissection technique. It should be mentioned that in the second case only two of the three regions could be detected by microFISH, since the size of the third was possibly below the sensitivity of microFISH.
Many additional marker chromosomes are very small ring chromosomes. According to the classical model, these ring chromosomes are considered to arise from chromosome breaks occurring on either side of the centromere and subsequent rejoining of the broken ends into a circular structure, while the segments distal to the breakpoints are lost. To generate small ring chromosomes, at least one of these two breaks is either at the centromere or adjacent to the centromere in the region containing alphoid repeats. The two cases described above and, in addition, our case with a discontinuous marker chromosome originating from two different regions of chromosome 18 clearly show that this assumption of formation of small rings is simplified. It is possible that many of the small ESACs identified only by FISH with centromere specific probes or chromosome specific libraries are inaccurately characterised. A correct description of ESACs is important since there may be recognisable clinical syndromes owing to the particular genetic content of the marker chromosomes. ESACs may contain genetic material located pericentromeric on the short or long arm or both but also, as shown in our case, may contain interstitial regions of the corresponding chromosome or even contain genetic material originating from other chromosomes. Therefore, different distinct syndromes may be associated with each group of morphologically similar markers. The precise characterisation of ESACs with this technology could provide the patient and the genetic counsellor with better predictive information on the phenotype of the carrier. MicroFISH would be a rapid method of investigating all cases in which the chromosomal origin could not be determined using alphoid repeats, and it could in addition provide a more accurate characterisation of all ESACs that have usually been studied only by FISH using centromere specific probe or chromosome specific libraries or both, including multicolour FISH approaches. ${ }^{9}$ MicroFISH provides a technique not only to identify the origin of marker chromosomes but also to detect the euchromatin involved and thus provide information on the composition of ESACs.

A more detailed description of small supernumerary marker chromosomes could also result in a better understanding of their formation. In our case we could show that the marker originated from two different regions of the normal maternally inherited chromosome 18 . After exclusion of a paracentric inversion or an intrachromosomal insertion of the long arm of chromosome 18 in the mother, at least four breaks are necessary to explain the formation of this ESAC, probably by the breakdown of a larger ring chromosome 18 until the stable form was generated. The breakpoint at $18 \mathrm{q} 21$ gives further evidence that this is a hot spot for breakpoints in complex chromosomal rearrangements. ${ }^{10}$

As surprising as these microFISH results may appear, the clinical findings are equally unexpected. Published cases with duplication of a similar chromosomal region display a more severe phenotype characterised by microcephaly, short stature, and more pronounced 
developmental delay. ${ }^{11}$ In contrast, our patient had normal measurements at birth as well as later in life and a global IQ of 76. Minor dysmorphic signs such as epicanthic folds, upward slanting palpebral fissures, widely spaced nipples, and short 5th fingers are present in our case as well as in most of the formerly reported cases. The less severe phenotype in our case could be explained by mosaicism and by the smaller size of the duplicated region.

In conclusion, since the great variability of phenotypes in patients with ESACs of the same chromosomal origin is probably the result of variations in their genetic content (in addition to the degree of mosaicism and the possibility of uniparental disomy), microFISH is a prerequisite for an accurate characterisation of small supernumerary marker chromosomes, as shown in our case with a discontinuous ESAC originating from two different regions of chromosome 18. Such a detailed characterisation and precise description of the genetic content is not possible with other molecular cytogenetic methods, namely FISH with centromere specific probes or chromosome specific libraries, including multicolour FISH approaches. The more accurate characterisation of ESACs may result in the description of different distinct syndromes associated with each group of morphologically similar but genetically different markers.
The authors are grateful to the parents of the proband and to Mrs Janine Steullet for skilful technical assistance. The study was supported by the Swiss National Foundation, grants No $32-56053.98,32-37798.93$ and 32.45604 .95 and grant no $71 \mathrm{P} 51778$.

1 Crolla JA. FISH and molecular studies of autosomal supernumerary marker chromosomes excluding those derived from chromosome 15. II. Review of the literature. Am $\mathfrak{F}$ Med Genet 1998;75:367-81.

2 Masuno M, Imaizumi K, Ishii T, Kimura J, Kuroki Y. Supernumerary ring chromosome 5 identified by FISH. Am $f$ Med Genet 1999;84:381.

3 Gardner RJM, Sutherland GR. Chromosome abnormalities and genetic counseling. Oxford: Oxford University Press, 1996:182-90.

4 Blennow E, Telenius H, Larsson C, et al. Complete characterization of a large marker chromosome by reverse and forward chromosome painting. Hum Genet 1992;90:371-4.

5 Senger G, Lüdecke HJ, Horsthemke B, Claussen U. Microdissection of banded human chromosomes. Hum Genet 1990;84:507-11

6 Lichter P, Ried T. Molecular analysis of chromosome aberrations. In situ hybridization. Methods Mol Biol 1994;29: 449-78.

7 Mitelman F, ed. ISCN. An international system for human cytogenetic nomenclature. Basel: Karger, 1995.

8 Fang YY, Eyre HJ, Bohlander SK, et al. Mechanisms of small ring formation suggested by the molecular characterization of two small accessory ring chromosomes derived from chromosome 4. Am f Hum Genet 1995;57:1137-42.

9 Haddad BR, Schröck E, Meck J, et al. Identification of de novo chromosomal markers and derivatives by spectral karyotyping. Hum Genet 1998;103:619-25.

10 Batanian JR, Eswara MS. De novo apparently balanced complex chromosome rearrangement (CCR) involving chromosomes 4, 18, and 21 in a girl with mental retardation: report and review. Am f Med Genet 1998;78: 44-51.

11 Schinzel A. Human cytogenetic database. Oxford: Oxford University Press, 1994. 